\title{
SIMULAÇÃO COMPUTACIONAL DE AGREGADOS DE POPG NA PRESENÇA DO PEPTÍ DEO ANTIMICROBIANO LL37
}

\author{
Yeny Y. P. Valencia ${ }^{1 *}$, Gabriel C. A. da Hora ${ }^{1}$, Thereza A. Soares ${ }^{1}$, \\ 1'Departamento de Química Fundamental, Centro de Ciências Exatas e da Natureza, UFPE \\ *yaneth.uq@gmail.com
}

\section{INTRODUÇÃO}

Os peptídeos antimicrobianos (AMPs) são compostos biológicos com atividade de amplo espectro contra bactérias, vírus e fungos. AMPs são moléculas anfipáticas com regiões hidrofóbicas e hidrofílicas (carregada positivamente). Em geral, a região hidrofílica pode iniciar a interação do peptídeo com a superfície bacteriana carregada negativamente e os grupos de cabeça negativamente carregados dos fosfolipídios. Já a parte hidrofóbica permitiria que os peptídeos penetrassem no interior da membrana com a possibilidade de ruptura de membranas lipídicas. (WU et al., 1999). Uma vez em contato com a membrana microbiana alvo, o peptídeo pode provocar a ruptura das células através de diversos mecanismos propostos.

Esses mecanismos podem ser classificados como: modelo barril, agregado, carpete e poro toroidal. Estudos recentes indicam que diferentes peptídeos penetram nas células alvo, podendo também interagir com outras membranas intracelulares. (BROWN; HANCOCK, 2006; WU et al., 1999)

A base molecular destes mecanismos é de grande interesse, mas nã o é completamente elucidado para vários AMPs. Acredita-se que diferenças na fluidez de membrana e composição lipídica interfiram na associação e ação dos AMPs às membranas alvo, além da estrutura de cada peptídeo, o potencial de interação e sua natureza catiônica e anfifílica (FEDER; NEHUSHTAI; MOR, 2001).

O LL-37 é um peptídeo antimicrobiano anfipático, com estrutura alfa-helicoidal em pH fisiológico, derivado de catelicidina encontrado em humanos. É formado por uma cadeia de 37 resíduos (LLGDF-FRKSK-EKIGK-EFKRI-VQRIK-DFLRN-LVPRT-ES) e faz parte da primeira linha de defesa contra infecção local e invasão sistêmica de patógenos em locais de inflamação. A pH fisiológico, 16 dos seus 37 resíduos são carregados, ou seja, 6 resíduos Lys e 5 Arg apresentam 11 cargas positivas, enquanto que 3 resíduos Glu e 2 Asp possuem 5 cargas negativas. A carga líquida resultante ao $\mathrm{pH}$ fisiológico é de + 6. (DÜRR; SUDHEENDRA; RAMAMOORTHY, 2006) Uma inspeção mais próxima do LL-37 revela que na região $\mathrm{N}$ terminal, as partes anfipáticas da hélice são menos perfeitamente formadas. Isso sugere que a $\mathrm{N}$-terminal é menos propícia para formar uma estrutura secundária em a-hélice, o que explica assim a proporção de apenas 20 a 30\% de estrutura em curva aleatória identificada por espectroscopia de dicroísmo circular. A região Cterminal forma uma a-hélice alifática, que é o principal padrão estrutural recorrente em todas os AMPs a-helicoidais conhecidos. Este padrão anfifílico não se estende por toda a molécula. Em vez disso, a região $\mathrm{N}$-terminal consiste em extensões de resíduos hidrofílicos e hidrofóbicos, que se aproximam para formar dois "ané is" ou "fitas" em torno do terminal N (assumindo que este terminal é de estrutura helicoidal a) (DÜRR; SUDHEENDRA; RAMAMOORTHY, 2006).

Dados preliminares por difração de raio-X de baixo ângulo (SAXS) sugerem que o LL37 desestabiliza bicamadas de POPG (1-Palmitoil2-Oleoil-sn-Glicero-3-[Fosfo-rac-(1-glicerol)]) e induz a formação de vesículas multi-lamelares com os peptídeos espacialmente localizados entre estas lamelas.

No presente projeto, pretendemos caracterizar o mecanismo molecular pelo qual o peptídeo antimicrobiano LL37 induz a desestabilização de agregados de POPG utilizando, para isso, o mé todo computacional de dinâmica molecular (MD). Membranas de POPG são comumente utilizadas como modelos experimentais representativos de células bacterianas, enquanto membranas de POPC (1-Palmitoil-2-Oleoil-sn-Glicero-3-Fosfatidilcolina) são utilizados como modelo representativo de hematócitos. Neste projeto, membranas de POPC são utilizadas como controle negativo dado que experimentalmente estas membranas não exibem desestabilização causada pelo LL37.

Foram realizadas simulações computacionais para vários sistemas contendo apenas POPG ou POPC, assim como para membranas de POPG e POPC em presença de múltiplas cópias do peptídeo LL37 em bicamadas de POPG e POPC. Foram simulados agregados lipí dicos organizados em bicamadas e vesículas (Tabela 1).

\section{MATERIAIS E MÉTODOS}

Simulações de dinâmica molecular com modelos coarse-grained (CG) foram realizadas para bicamadas e vesículas de POPG e POPC na presença e ausência do peptídeo antimicrobiano LL37. Os sistemas foram solvatados com moléculas de água CG e adicionados $150 \mathrm{mM}$ de $\mathrm{NaCl}$ para reprodução da concentração fisiológica. Os sistemas simulados são mostrados na Tabela 1.

\begin{tabular}{|c|c|c|c|c|}
\hline \multirow[t]{2}{*}{ SISTEMA } & \multicolumn{3}{|c|}{ NÚMERO DE MOLÉCULAS } & \multirow[t]{2}{*}{$\begin{array}{l}\text { TEMPO DA } \\
\text { SIMULAÇÅO }\end{array}$} \\
\hline & LIPIDEOS & PEPTIDEOS & IONS & \\
\hline POPG_BICAMADA & 520 & 0 & $520 \mathrm{Na}^{+}$ & $300 \mathrm{~ns}$ \\
\hline POPG_VESÍCULA & 1000 & 0 & \multicolumn{2}{|c|}{ Em andamento } \\
\hline POPC_BICAMADA & 520 & 0 & $57 \mathrm{Na}^{+} / 57 \mathrm{Cl}^{-}$ & $300 \mathrm{~ns}$ \\
\hline POPC_VESÍCULA & 950 & 0 & \multicolumn{2}{|c|}{ Em andamento } \\
\hline POPG_BICAMADA_LL37 & 520 & 10 & $520 \mathrm{Na}^{+} / 60 \mathrm{Cl}^{-}$ & $\begin{array}{l}\text { 300ns } \\
300 \text { us }\end{array}$ \\
\hline POPG_VESÍCULA_LL37 & 1000 & $\cdots$ & \multicolumn{2}{|c|}{ Em andamento } \\
\hline POPC_BICAMADA_LL37 & 520 & 10 & $57 \mathrm{Na}^{+} / 117 \mathrm{Cl}^{-}$ & $300 \mathrm{~ns}$ \\
\hline POPC_VESÍCULA_LL37 & 950 & $\cdots$ & \multicolumn{2}{|c|}{ Em andamento } \\
\hline
\end{tabular}


O campo de força MARTINI (MARRINK et al., 2007; MARRINK; DE VRIES; MARK, 2004)foi usado em conjunto com o pacote de software GROMACS v4.6.7. (HESS et al., 2008)

O MARTINI é um campo de força para simulações de dinâmica molecular de biomoléculas coarse-grained (CG) parametrizado de forma sistemática. Na resolução $\mathrm{CG}$, vários átomos são combinados em grupos funcionais conhecidos como super-átomos como se mostra na figura 1, diminuindo o número de partículas no sistema e suavizando a paisagem energética o que permite passos de tempo de integração maiores e muitas vezes leva a uma dinâmica efetiva mais rápida em comparação as simulações com todos os átomos. (CARPENTER et al., 2017). As simulações com modelos CG serão mapeadas de volta para modelos atômicos e comparados com medidas experimentais de SAXS.
A.

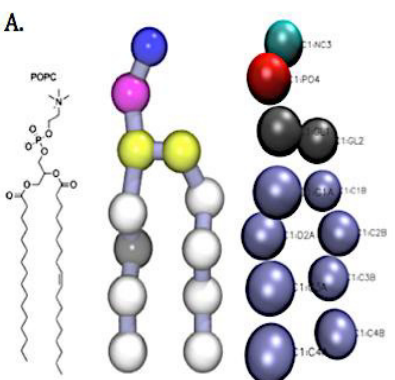

B.

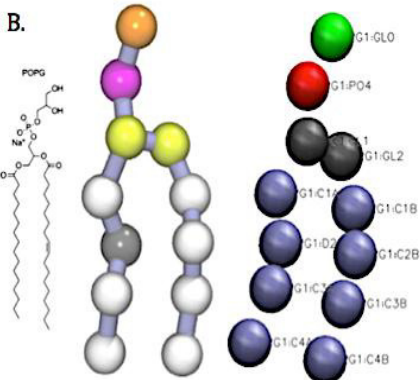

Figura 1. Representação esquemática das estruturas usadas neste trabalho. A. Modelo atomístico e coarse-grained do POPC. B. Modelo atomístico e coarse-grained do POPG. (Fonte: Coarse Grain Forcefield for Biomolecules, Martini).

As bicamadas de membrana CG foram construídas com o script INSANE (WASSENAAR et al., 2015) e as vesículas CG com o software PACKMOL. (MARTíNEZ et al., 2009) O programa PACKMOL cria um ponto inicial para as simulações de dinâmica molecular através do empacotamento de moléculas em regiões definidas do espaço. Esse empacotamento garante que as interações repulsivas de curto alcance não interrompem as simulações. (MARTíNEZ et al., 2009) Para a criação da vesícula foi necessário fornecer as coordenadas nos eixos $\mathrm{X}, \mathrm{Y}$ e $\mathrm{Z}$ de um determinado número de super-átomos (coarse-grained), o número de moléculas de água e o tamanho da caixa, respeitando o ordenamento espacial.

Os sistemas foram submetidas a uma otimização de energia e neutralizados através da adição de contra-íons $\mathrm{Na}^{+}$(apenas para POPG), seguido por uma segunda rodada de minimização de energia (5000 passos), equilibração de energia e dinâmica durante 300 ns sob condições NpT (temperatura e pressão constante). No caso dos sistemas de POPG e POPC com peptídeo LL37 foram necessários a adição de íons $\mathrm{Cl}^{-}$adicionais para neutralizar a carga total dos peptídeos. O termostato de Berendsen (DA HORA, 2016) foi usado para manter o sistema na temperatura de $305 \mathrm{~K}$. A pressã o foi mantida em 1 bar através do acoplamento fraco das coordenadas das partículas e das dimensões da caixa no plano $x y$ e ao longo do eixo $z$ por meio de escalonamento de coordenadas semi-isotrópicas com um tempo de relaxamento de 0,1 ps e uma compressibilidade de $4,5 \times 10^{-5}\left(\mathrm{~kJ} \mathrm{~mol}^{-1} \mathrm{~nm}^{-3}\right)^{-1}$, conforme apropriado para a água.(DA HORA et al., 2016) Por fim, realizamos análises e discutimos propriedades estruturais derivadas desses simulações.

\section{RESULTADOS E DISCUSSÃO}

Estudo das bicamadas de POPG e POPC na ausência do peptídeo antimicrobiano LL37.

Medidas experimentais de SAXS indicam que o peptídeo antimicrobiano LL37 desestabiliza os agregados de POPG. Portanto, estão sendo realizadas diferentes simulações exploratórias para reproduzir as tendências experimentais e estabelecer o mecanismo apropriado. Até o momento foram simulados os sistemas mostrados na tabela 1 de bicamadas de POPG e POPC, como mostradas na figura 2.
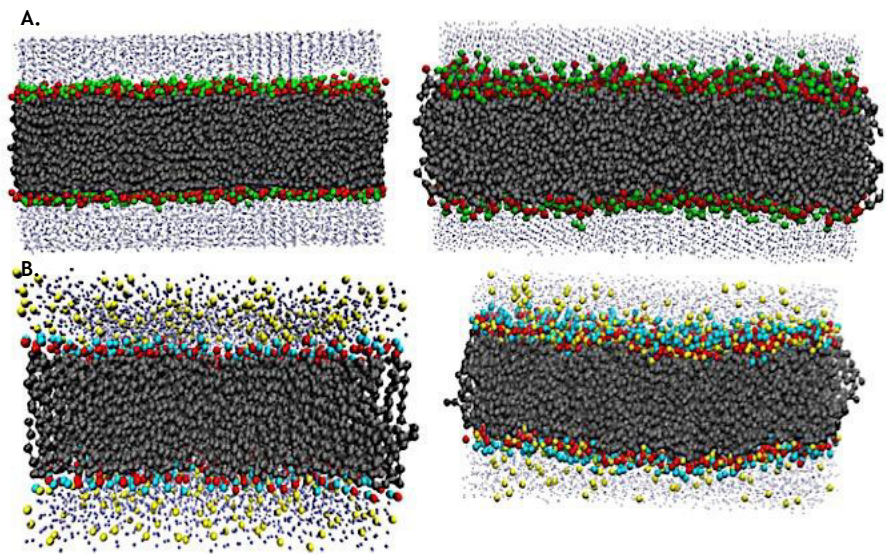

Figura 2. Representação dos sistemas de bicamadas de POPC e POPG. A. Na esquerda sistema de bicamada de POPC durante a minimização de energia e na direita depois da simulação de dinâmica molecular para 300 ns. As moléculas da água são mostradas em cinza, os fosfatos em vermelho e o NC3 em verde. B. Na esquerda sistema de bicamada de POPG durante a minimização de energia e na direita depois da simulação de dinâmica molecular para 300 ns. As moléculas da água são mostradas em cinza, os íons sódio em amarelo, os fosfatos em vermelho e o GL0 em celeste. (Fonte: elaboração própria).

Essas simulações são importantes porque fornecem uma estimativa da convergência de propriedades estruturais a partir da comparaçã o com os valores experimentais medidos para as bicamadas puras, além de garantir que as bicamadas tenham sido adequadamente equilibradas antes da adição do peptídeo antimicrobiano. Para verificar isso, foram realizadas analises de área por cabeça de lipí dio e número de densidade. A.
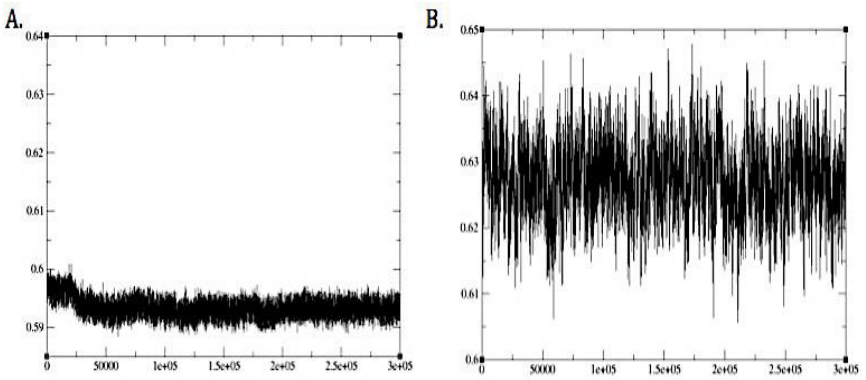

Figura 3. Área por lipídio. A. Bicamada de POPC. B. Bicamada de POPG.

Os valores calculados da área por cabeça correspondem aos valores medidos experimentalmente $0.54-0.68 \mathrm{~nm}^{2}(\mathrm{POPC})$ e $0.55-0.67 \mathrm{~nm}^{2}$ (POPG).(PABST et al., 2000)
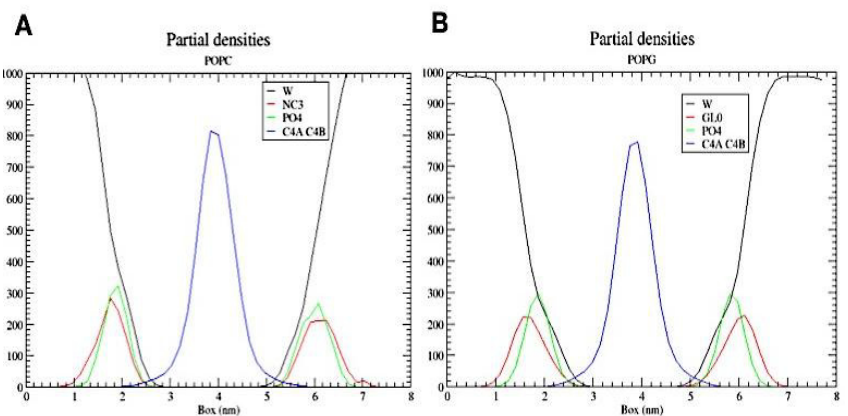

Figura 4. Densidade parcial. A. Bicamada de POPC. B. Bicamada de POPG.

Os sistemas apresentam uma estrutura lamelar similar ao padrão estrutural de bicamada: as moléculas de agua estão confinadas na região hidrofílica formadas pelos grupamentos de glicerol e grupos fosfato, os quais constituem as cabeças dos lipídios; a região 
hidrofóbica também é bem definida em todos os casos: os átomos terminais das cadeias, C4A e C4B, estão afastados das moléculas de água e dispostos ao longo da região central da caixa. Em ambos os casos, a caixa possui uma distribuição simétrica característica de sistemas de bicamadas.

O comportamento da membrana de POPC e POPG na presença do peptídeo antimicrobiano LL37

Foram realizadas também simulações na presença de peptídeo antimicrobiano, após a dinâmica molecular dos sistemas de POPG e POPC. A seguir, apresentamos os dados obtidos para a interação do peptídeo antimicrobiano LL37 com a bicamada de POPG e POPC.
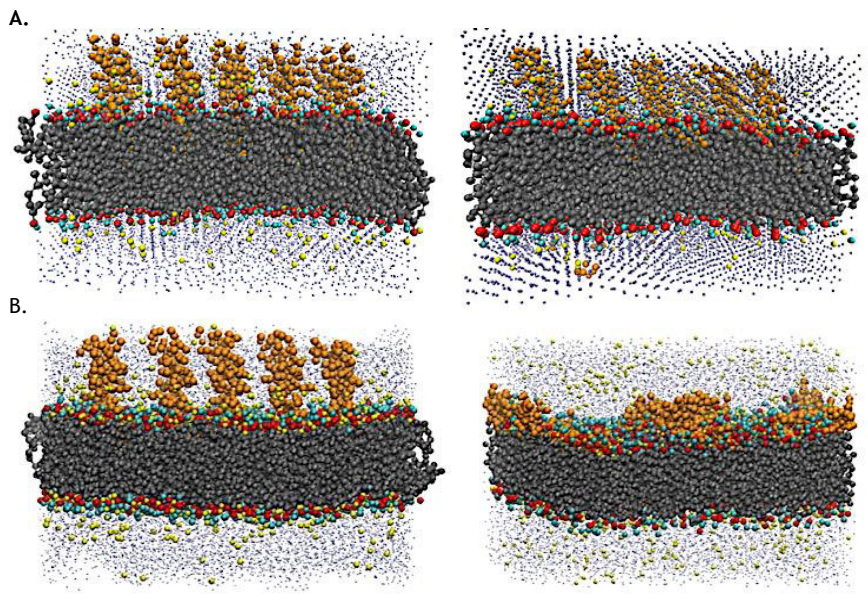

Figura 5. Representação dos sistemas de bicamadas de POPC, POPG com o peptídeo antimicrobiano LL37. A. Configuração inicial do sistema de bicamada de POPC-LL37 no iní cio (esquerda) e após 300 ns (direita) de simulação B. Configuração inicial do sistema de bicamada de POPG-LL37 no início (esquerda) e após 300 ns (direita) de simulação. As moléculas da água são mostradas em cinza, os íons sódio em amarelo, os fosfatos em vermelho e o GLO/NC3 em celeste. (Fonte: elaboração própria).

O sistema de bicamada POPC-LL37 não exibe grandes modificações, e os peptídeos permanecem em conformação similar a inicial. na membrana de POPG, o efeito da interação do aglomerado de peptídeos acentuado, provocando grandes deformações na bicamada lipídica. Foram também realizadas analises de área por cabeça de lipídio e número de densidade parcial que são mostrados nas figuras 6 e 7 .

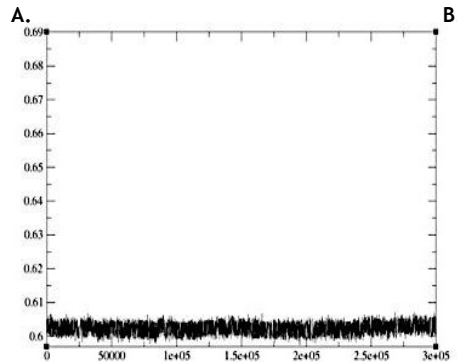

B.

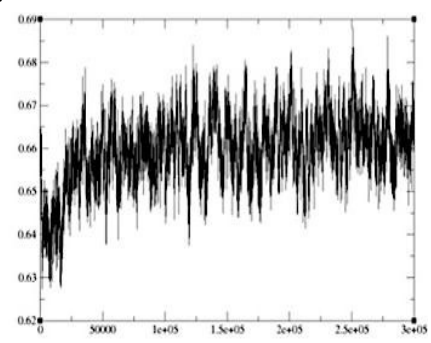

Figura 6. Área por lipídio. A. Bicamada de POPC. B. Bicamada de POPG.

A.

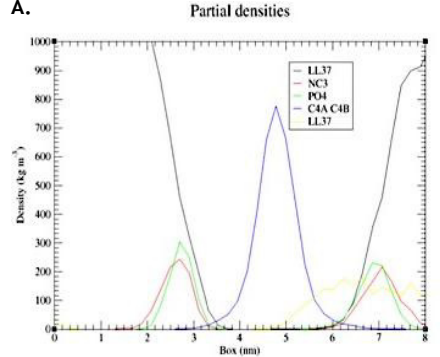

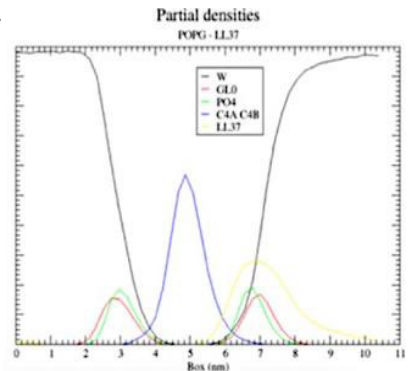

Figura 7. Área por lipídio. A. Bicamada de POPC. B. Bicamada de POPG.

Os gráficos mostram que existe uma ligeira variação da área por cabeça na presença de LL37, assim como um pequeno deslocamento na distribuição da membrana na caixa de simulação.

\section{CONCLUSÕES}

As presentes simulações CG MD são muito curtas para permitir conclusões finais. No entanto, identificamos parâmetros adequados e ferramentas de software para preparar e simular sistemas moleculares representativos do fenômeno molecular de interesse, que é o efeito do LL37 em agregados POPG e POPC. Atualmente estamos preparando a mesma configuração para modelos mais complexos e, portanto, representativos de membranas bacterianas e mamíferas.

\section{REFERÊNCIAS}

BROWN, K. L.; HANCOCK, R. E. Cationic host defense (antimicrobial) peptides. Current Opinion in Immunology, [s. l.], v. $18, \quad$ n. 1 , p. 24-30, 2006. Disponível em: <http://linkinghub.elsevier.com/retrieve/pii/S0952791505001998> CARPENTER, T. S. et al. Computational Lipidomics of the Neuronal Plasma Membrane. [s. l.], p. 2271-2280, 2017.

rias Compostas por POPC:POPG. [s. l.], p. 147, 2016.

DA HORA, G. C. A. et al. Membrane negative curvature induced by a hybrid peptide from pediocin PA-1 and plantaricin 149 as revealed by atomistic molecular dynamics simulations. Soft Matter, [s. l.], v. 12, n. 43, p. 8884-8898, 2016. Disponível em: <http:/ / xlink.rsc.org/?DOI=C6SM01714B >

DÜRR, U. H. N.; SUDHEENDRA, U. S.; RAMAMOORTHY, A. LL-37, the only human member of the cathelicidin family of antimicrobial peptides. Biochimica et Biophysica Acta (BBA) - Biomembranes, [s. l.], v. 1758, n. 9, p. 1408-1425, 2006. Disponível em: <http://linkinghub.elsevier.com/retrieve/pii/S000527360600126X> FEDER, R.; NEHUSHTAI, R.; MOR, A. Affinity driven molecular transfer from erythrocyte membrane to target cells. Peptides, [s. l.], v. 22, n. 10, p. 1683-1690, 2001. Disponivel em: <http://linkinghub.elsevier.com/retrieve/pii/S0196978101005046> HESS, B. et al. GROMACS 4: Algorithms for Highly Efficient, LoadBalanced, and Scalable Molecular Simulation. Journal of Chemical Theory and Computation, [s. l.], v. 4, n. 3, p. 435-447, 2008. Disponível em: <http://pubs.acs.org/doi/abs/10.1021/ct700301q > MARRINK, S. J. et al. The MARTINI Force Field: Coarse Grained Model for Biomolecular Simulations. The Journal of Physical Chemistry B, [s. l.], v. 111, n. 27, p. 7812-7824, 2007. Disponível em: <http://pubs.acs.org/doi/abs/10.1021/jp071097f >

MARRINK, S. J.; DE VRIES, A. H.; MARK, A. E. Coarse Grained Model for Semiquantitative Lipid Simulations. The Journal of Physical Chemistry B, [s. l.], v. 108, n. 2, p. 750-760, 2004. Disponível em: <http://pubs.acs.org/doi/abs/10.1021/jp036508g>

MARTÍNEZ, L. et al. PACKMOL: A package for building initial configurations for molecular dynamics simulations. Journal of Computational Chemistry, [s. l.], v. 30, n. 13, p. 2157-2164, 2009. Disponível em: <http://doi.wiley.com/10.1002/jcc.21224>

PABST, G. et al. Structural information from multilamellar liposomes at full hydration: full q-range fitting with high quality $\mathrm{x}$ ray data. Physical review. E, Statistical physics, plasmas, fluids, and related interdisciplinary topics, [s. l.], v. 62 , n. 3 Pt B, p. 4000-9, 2000.2 Disponível em: <http://www.ncbi.nlm.nih.gov/pubmed/11088921>

WASSENAAR, T. A. et al. Computational Lipidomics with insane : A Versatile Tool for Generating Custom Membranes for Molecular Simulations. Journal of Chemical Theory and Computation, [s. l.], v. 11, n. 5, p. 2144-2155, 2015. Disponível em: <http://pubs.acs.org/doi/abs/10.1021/acs.jctc.5b00209>

WU, M. et al. Mechanism of Interaction of Different Classes of Cationic Antimicrobial Peptides with Planar Bilayers and with the Cytoplasmic Membrane of Escherichia coli $\dagger$. Biochemistry, [s. l.], v. $38, \quad$ n. $22, \quad$ p. $7235-7242,1999$. Disponível em: <http://pubs.acs.org/doi/abs/10.1021/bi9826299> 